\title{
Estructura de la vegetación leñosa en tres áreas con Selva Baja Caducifolia en el Istmo-Costa de Oaxaca, México
}

\author{
Marisa Silva-Aparicio ${ }^{1}$, Adriana E. Castro-Ramírez ${ }^{1}$, Gonzalo Castillo-Campos ${ }^{2}$ \\ \& Hugo Perales Rivera ${ }^{1}$ \\ 1. Departamento de Agricultura, Sociedad y Ambiente, El Colegio de la Frontera Sur, San Cristóbal de las Casas, \\ Chiapas, 29290, México; *masilva@ecosur.edu.mx, castroadriana3@gmail.com; hperales@ecosur.mx \\ 2. Departamento de Biodiversidad y Sistemática, Instituto de Ecología, A.C. Xalapa, Veracruz, México; \\ gonzalo.castillo@inecol.mx
}

Recibido 08-VIII-2017. Corregido 10-I-2018. Aceptado 02-II-2018.

\begin{abstract}
Woody vegetation structure in three areas with Tropical Dry Forest in the Isthmus-Coastal of Oaxaca, Mexico. Tropical dry forest (TDF) is important due to its biodiversity. In the Isthmus-Coastal region of the state of Oaxaca, in Southeast Mexico, there are many areas of TDF with varying categories of legal protection (1) Protected Natural Areas (PNA), (2) Voluntarily Designated Conservation Areas (VDCA) and (3) Areas of Common Use (ACU). In each of these, tree vegetation structure was analysed, recording plants with a DBH $\geq 2.5 \mathrm{~cm}$ and sampling 15 plots of $100 \mathrm{~m}^{2}$. Observed richness was 90 species, belonging to 36 families and corresponding to $80 \%$ (Jackknife) and $85 \%$ (ICE) of expected richness. A cluster analysis identified two groups with $90 \%$ dissimilitude: the first (G1) included all the sampled plots in the PNA; and the second (G2), grouped together those from the VDCA and the ACU. The Shannon, Simpson dominance and Jost true diversity indices for $\mathrm{G} 1$ were $\mathrm{H}^{\prime}=2.43, \mathrm{D}=0.21$ and ${ }^{\mathrm{q}} \mathrm{D}=11$ and for $\mathrm{G} 2, \mathrm{H}^{\prime}=2.76, \mathrm{D}=0.28$ and ${ }^{\mathrm{9}} \mathrm{D}=15$ respectively, with no differences between groups $(\mathrm{P}<0.05)$. The species that presented the highest relative importance value (RIV) in both groups, corresponded to secondary vegetation. Species density of G1 was $1829 \mathrm{ind} / \mathrm{ha}$ and $2370 \mathrm{ind} / \mathrm{ha}$ for G2. Mean height $(6.8 \mathrm{~m})$ and basal area $\left(19.7 \mathrm{~m}^{2} / \mathrm{ha}\right)$ of $\mathrm{G} 1$ did not vary significantly $(\mathrm{P}<0.05)$ when compared to G2 $\left(7.9 \mathrm{~m} \mathrm{y} 22.1 \mathrm{~m}^{2} / \mathrm{ha}\right)$. The VDCA and ACU presented similar composition and structure, differing from the PNA. Continual monitoring of the TDF is suggested. Rev. Biol. Trop. 66(2): 863-879. Epub 2018 June 01.
\end{abstract}

Key words: Natural Protected Areas, areas of common use, voluntarily designated conservation areas, tropical dry forest, species diversity, floristic similitude.

El bosque tropical seco se define como aquel que posee un dosel cerrado (distinguiéndolo de la sabana, que es más abierto), crece en suelos fértiles, con precipitaciones inferiores a $\sim 1800 \mathrm{~mm}$ anuales, pero de tres a seis meses solo recibe $100 \mathrm{~mm}$ mensuales, periodo en que la vegetación pierde su follaje (Banda et al., 2016). Actualmente ocupa un área de $1048700 \mathrm{~km}^{2}$, de los cuales $54.2 \%$ se encuentran en América del Sur, $12.5 \%$ en América del Norte y Central, $13.1 \%$ en África, 16.4 $\%$ en Eurasia y $3.8 \%$ en Australasia y Asia Sudoriental (Miles et al., 2006). En México, este ecosistema es conocido como Selva Baja
Caducifolia (SBC) (Miranda \& Hernández, 1963) o Bosque Tropical Caducifolio (Rzedowski, 2006) y es uno de los mejor representados. Según Rzedowski (1991), ocupa entre el $11.26 \%$ y $17 \%$ del territorio nacional; Rincón, Álvarez, González, Huante y Hernández (1999) estimaron el $12.36 \%$; e INEGI (2013) el 9.0\%. Rzedowski (2006) señala que el número de especies referido para este ecosistema asciende a 6000 (20\% del total de la flora nacional), Villaseñor (2016) indica 3225; el porcentaje de especies endémicas se encuentra entre el 40 \% (Rzedowski, 1991) у 60 \% (Trejo, 1999). A pesar de la importancia que representa la SBC, 
la deforestación (tasa anual de 0.6) (AguilarMartínez \& Arriaga, 2000) ha llevado a la pérdida del $50 \%$ del área cubierta originalmente por este ecosistema (Trejo, 2005).

Oaxaca es una de las entidades con diversidad biológica más alta de México, comparable con la observada en los estados de Chiapas y Veracruz (Meave, Romero-Romero, Salas-Morales, Pérez-García, \& Gallardo-Cruz, 2012). La vegetación predominante es SBC, que se distribuye de manera discontinua al suroeste del estado (colindando con Guerrero), en las regiones Cañada, Valles Centrales, vertiente del río Tehuantepec, en el Istmo de Tehuantepec y Costa (Meave et al., 2012). La región de La Cañada ha sido más estudiada, debido a que ahí se sitúa la Reserva de la Biosfera Tehuacán-Cuicatlán. En el resto del estado de Oaxaca el trabajo ecológico y florístico es aún incompleto (Trejo \& Dirzo, 2000; yet information about its conservation situation is scarce. This study analyzes the loss of this forest at the national level, comparing its potential coverage with that of the early 1990s; and at the local, using a time-series of the potential vegetation and coverage in 1973 and 1989 in the state of Morelos (central MexicoGallardo-Cruz, Meave, \& Pérez-García, 2005; Meave et al., 2012, Cortés-Marcial $\&$ Briones-Salas, 2014); lo que es preocupante, por la disminución de la cubierta vegetal y la falta de registros de la biodiversidad que habita en estos ecosistemas (Dirzo, 1990; Trejo, 2005; Meave et al., 2012).

Se estima que la tercera parte de la flora del estado Oaxaca (3 000 especies) corresponde a SBC (Meave et al., 2012). Su estructura, composición florística y procesos dinámicos varían en relación con la disponibilidad de agua a nivel local y regional (Trejo \& Dirzo 2002; Gallardo-Cruz, Pérez-García \& Meave, 2009). De igual manera la variaciones edáficas grandes permiten la coexistencia de diversas formaciones vegetales en el mismo paisaje (Gallardo-Cruz, Meave, Pérez-García, \& Hernández-Stefanoni, 2010). Trejo (2010) menciona que en el piedemonte de los cerros o en las cañadas las mismas especies alcanzan tallas mayores y tienden a ser más perennifolias que en las laderas altas o en las cimas.

En México, una de las estrategias para mitigar el deterioro y propiciar la conservación de los recursos naturales (incluida la SBC) ha sido el incremento de las Áreas Naturales Protegidas (ANP) (Bertzky et al., 2012; SEMARNAT, 2016). Actualmente hay 182 ANP en México (CONANP, 2015), de las cuales sólo ocho se ubican en Oaxaca y la SBC está presente únicamente en la Reserva de la Biosfera Tehuacán-Cuicatlán, Monumento Natural Yagul y el Parque Nacional Huatulco.

El limitado número de ANP en Oaxaca se debe, posiblemente, a la constante lucha de sus pobladores por su autonomía, que incluye el manejo de los recursos naturales, por lo que tienen una larga tradición en la gestión colectiva de estos (Merino, 2008). En el año 2002 surgió una nueva modalidad de ANP denominada Área Destinada Voluntariamente para la Conservación (ADVC) (CONANP, 2015), que corresponde a zonas de conservación certificadas por la Comisión Nacional de Áreas Naturales Protegidas (CONANP), pero gestionadas por sus propietarios (individual o colectivo). Con esta propuesta, se han establecido 381 sitios protegidos en todo el país, 138 de ellos se localizan en Oaxaca (CONANP, 2015), de los cuales 23 albergan SBC. El aumento del número de áreas protegidas (incluyendo las ADVC) es significativa, no obstante se considera que la superficie es aún insuficiente para conservar la SBC (Meave et al., 2012). Es necesario incluir en la estrategia de conservación a las áreas de uso común (ADUC), puesto que alrededor de $60 \%$ de los bosques de México son propiedad de ejidos (29 441 títulos) o comunidades (2 344 títulos) (Madrid, Núñez, Quiroz, \& Rodríguez, 2009; Reyes et al., 2012). En el caso de Oaxaca, la propiedad social alcanza el $78 \%$ de la superficie estatal, distribuida en 1564 núcleos agrarios (RAN, 2016), con una porción considerable de SBC administrada por poblaciones locales, por lo que podrían ser de gran relevancia para la preservación de su biodiversidad (Meave et al., 2012). 
Las ANP, ADVC y ADUC albergan parte de la diversidad biológica; no obstante, los estudios sobre la SBC en la región IstmoCosta de Oaxaca no se han enfocado a las áreas señaladas, ya que se desarrollaron en espacios relativamente conservados pero, en su mayoría, sin pertenecer a alguna categoría de conservación (Gallardo-Cruz et al., 2005). Así, resulta interesante la exploración cuantitativa para determinar la condición en que se encuentra actualmente la SBC, en estos tres tipos de áreas. Este estudio se propuso determinar la estructura (área basal, DAP; densidad de dos categorías de DAP, individuos/ha; altura, m; valor de importancia relativa, VIR) y diversidad de las especies leñosas (riqueza, $\mathrm{S}$; diversidad verdadera, ${ }^{\mathrm{a}} \mathrm{D}$; índice de Shannon, H'; e índice de Simpson, D) de SBC que se desarrollan en un ANP, el Parque Nacional Huatulco, además de exponer su similitud con un ADVC con 10 años de certificación y una ADUC, ambas situadas en el núcleo agrario de San Pedro Huamelula en la región Istmo-Costa de Oaxaca.

\section{MATERIALES Y MÉTODOS}

Área de estudio: El núcleo agrario de San Pedro Huamelula se ubica en la región del Istmo de Tehuantepec (Fig. 1); posee 51000 ha, de las cuales 10000 ha forman parte del ADVC denominada "Mascalco", certificada en el año 2006. El resto de la superficie está registrada como área de uso común (ADUC), en ella se localizan los asentamientos humanos y se desarrollan labores agropecuarias, obtención

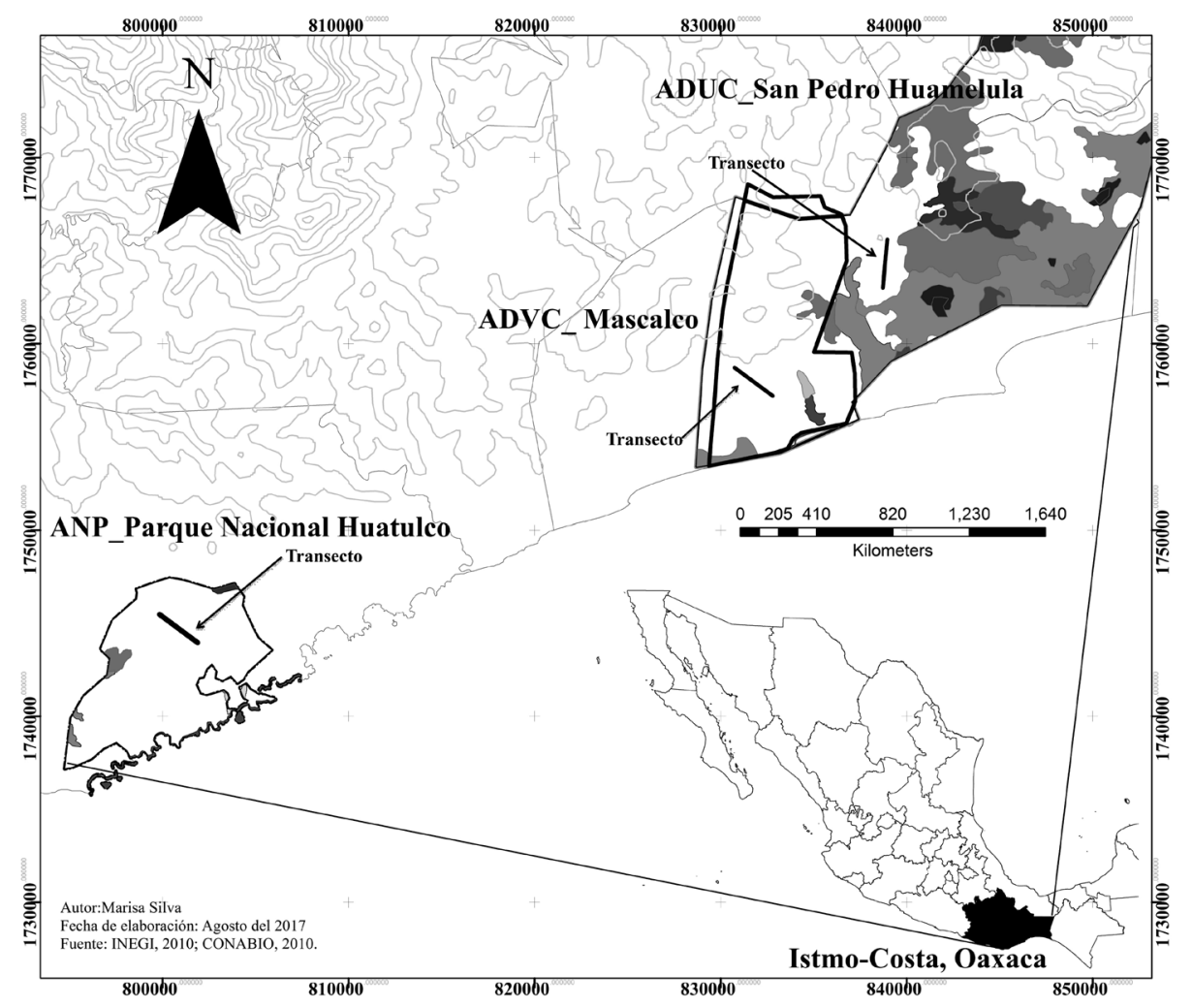

Fig. 1. Transectos en las áreas de estudio: Área Destinada Voluntariamente para la Conservación (ADVC), Área de Uso Común (ADUC) y Área Natural Protegida (ANP) en el Istmo-Costa de Oaxaca, México.

Fig. 1. Transects in the study areas: Voluntary Conservation Area (ADVC), Common Use Area (ADUC) and Protected Natural Area (ANP) in the Istmo-Costa de Oaxaca, Mexico. 
de madera y leña, entre otras actividades; estos espacios tienen reglas de uso establecidas por las asambleas, con ellas controlan el aprovechamiento de los recursos naturales. En el ADVC las actividades mencionadas están prohibidas (Comunicación personal: Comisariado de bienes comunales, 2016).

El Parque Nacional Huatulco fue decretado en 1998 y cuenta con una superficie total de 11 890ha (INE, 2000). Según el plan de manejo (CONANP, 2003), el área incluye las siguientes zonas: a) de protección, donde sólo se puede realizar investigación, vigilancia biológica y educación ambiental; b) de uso restringido, las actividades permitidas están bajo estricto control; c) de uso tradicional, son superficies donde los recursos naturales se aprovechan de manera tradicional y continua, sin ocasionar alteraciones significativas en el ecosistema; d) de uso sostenible, los recursos pueden aprovecharse bajo un esquema de desarrollo sustentable; y e) de recuperación, donde los recursos naturales fueron severamente modificados y están bajo programas de recuperación.

Selección de sitios: El trabajo se realizó entre febrero 2015 y marzo 2016. Por su extensión, las ANP, ADVC y ADUC presentan distintas características ambientales (Cuadro 1), por lo que la selección de sitios de muestreo incluyó la elaboración de una clasificación de la vegetación no supervisada (Chuvieco, 2000) a partir de imágenes de satélite Landsat ETM+ (obtenidas de earthexplorer.usgs.gov) de enero 2015, utilizando los programas MultiSpecW32 y ArcGIS 10.0. Las clases de vegetación establecidas se verificaron a través de un muestreo estratificado. El muestreo en el ANP y ADVC fue en sitios con acceso restringido y en el ADUC donde la intervención de los pobladores locales fuera casi mínima (determinada con ayuda de los guías de campo).

Caracterización de la vegetación de leñosas: En los sitios seleccionados de cada área se trazaron transectos de alrededor de $3 \mathrm{~km}$, las coordenadas iniciales y el rumbo se determinaron previamente con los mapas elaborados y los recorridos de campo. Sobre esta línea se establecieron 15 cuadros o parcelas de $100 \mathrm{~m}^{2}$ (Palacios-Wassenaar, Castillo-Campos, Vázquez-Torres \& Del Amo-Rodríguez, 2014) En cada una se anotó el número de individuos por especie leñosa (arbusto, árbol, arborescente

\section{CUADRO 1}

Características físico-ambientales de San Pedro Huamelula y del Parque Nacional de Huatulco, en Oaxaca

TABLE 1

Physical-environmental characteristics of San Pedro Huamelula and the Huatulco National Park in Oaxaca

\begin{tabular}{|c|c|c|}
\hline & San Pedro Huamelula & Parque Nacional Huatulco \\
\hline Superficie (ha) & 51050.13 & 11890.98 (6 374.98 terrestres $)$ \\
\hline Fisiografía & $\begin{array}{l}\text { Provincia: Sierra Madre del Sur } \\
\text { Subprovincia: Sierra Costas del Sur (sierra baja } \\
\text { compleja, lomerío con llanuras, llanura costera con } \\
\text { lomerío y sierra alta compleja). }\end{array}$ & $\begin{array}{l}\text { Provincia: Sierra Madre del Sur } \\
\text { Subprovincia: Sierra Costas del Sur } \\
\text { (montañas medianas, lomeríos complejos y } \\
\text { llanuras fluviales). }\end{array}$ \\
\hline Suelos & $\begin{array}{l}\text { Cambisol, Phaeozem, Regosol, Leptosol, Luvisol, } \\
\text { Fluvisol, Litosol, Gleysol, Arenosol y Vertisol. }\end{array}$ & Regosol y Litosol. \\
\hline Clima & $\begin{array}{l}\text { Temperatura } 18-28{ }^{\circ} \mathrm{C} \text {. Precipitación } 700-1000 \mathrm{~mm} \text {. } \\
\text { Clima, cálido subhúmedo con lluvias en verano } \\
(98.11 \%) \text { y semicálido subhúmedo con lluvias } \\
\text { en verano }(1.89 \%) \text {. }\end{array}$ & $\begin{array}{l}\text { Temperatura } 18-28{ }^{\circ} \mathrm{C} \text {. Precipitación } \\
1000-1500 \mathrm{~mm} \text {. Cálido subhúmedo con } \\
\text { lluvias en verano. }\end{array}$ \\
\hline Vegetación & $\begin{array}{l}\text { Selva baja caducifolia, Dunas costeras, Riparia, Sabana, } \\
\text { Manglar. }\end{array}$ & $\begin{array}{l}\text { Selva baja caducifolia, Dunas costeras, } \\
\text { Riparia, Sabana, Manglar. }\end{array}$ \\
\hline
\end{tabular}

Fuente: Información tomada de CONANP (2015) e INEGI (2015). 
o bejuco) su diámetro a la altura del pecho (DAP a $1.30 \mathrm{~m}$ ) $\geq 2.5 \mathrm{~cm}$ y su altura total (utilizando un clinómetro Suunto) (Chave, 2005). Se recolectó un ejemplar de cada espécimen. Adicionalmente, en tres cuadros de $4 \mathrm{~m}^{2}$ cada uno, distribuidos al azar en el interior de las parcelas de $100 \mathrm{~m}^{2}$, se llevó a cabo el recuento de individuos con $10 \mathrm{~cm}$ de altura y DAP $<2.5 \mathrm{~cm}$ (juveniles de las especies leñosas, además de bejucos).

Composición florística: Los especímenes en su mayoría se identificaron a especie en los herbarios ECOSUR-SCLC (El Colegio de la Frontera Sur, Chiapas) y SERBO (de la Sociedad para el Estudio de los Recursos Bióticos de Oaxaca A.C.), utilizando claves dicotómicas de floras afines (INECOL, 2016; Jiménez-Ramírez \& Martínez-Gordillo, 2004; Pennington \& Sarukhán 2005; Barajas-Morales \& PérezJiménez, 1990) y compararon con material herborizado determinado por especialistas de los distintos grupos de plantas depositados en dichos herbarios. Los ejemplares botánicos llevan la numeración de Silva-Aparicio 1 hasta 263 y están depositados en la colección del herbario ECOSUR-SCLC.

La nomenclatura utilizada para clasificar la vegetación presente en el área de estudio es de Miranda y Hernández-X. (1963); los géneros, especies y autoridades taxonómicas fueron revisados con la base de datos VAST del Missouri Botanical Garden (W3 TROPICOS, 2016).

El esfuerzo de muestreo se evaluó mediante la curva de acumulación de especies por área, con el programa EstimateS versión 9.1 (Colwell, 2013; Magurran, 2004). Se utilizaron los estimadores Chao 2, ICE y Jackknife 1 ya que, de acuerdo con autores como Chazdon, Colwell, Denslow y Guariguanta (1998) y López-Gómez y Williams-Linera (2006), son los adecuados para especies de plantas leñosas.

Con base en la lista florística del inventario de una superficie de $4500 \mathrm{~m}^{2}$ de las 45 parcelas de cada una de las áreas de uso (ADUC, ADVC y ANP), se elaboró una matriz de presencia/ausencia para evaluar la similitud de los grupos formados mediante el índice de Jaccard (Sneath \& Sokal, 1973); utilizando la media aritmética no ponderada (UPGMA). El dendrograma se generó con el programa Multi Variate Statistical Package (MVSP), versión 3.22 (Kovach, 2013). Se consideró $0=100 \%$ de disimilitud y $1=100 \%$ de similitud, usando la presencia-ausencia de las especies inventariadas en las parcelas.

Para la diversidad alfa se estimó la riqueza de especies (S) de la comunidad de leñosas, además de los índices de Shannon $\left(\mathrm{H}^{\prime}\right)$, Simpson $(\mathrm{D}=$ dominancia $)$ y diversidad verdadera de Jost $\left({ }^{\mathrm{q}} \mathrm{D}=\left(\sum_{\mathrm{i}-1} \mathrm{~s} p i^{q}\right)^{1 /(1-\mathrm{q})}\right)$. Para calcular la diversidad beta $(\beta)$ entre áreas y grupos se utilizó el índice de Jaccard ( $\mathrm{I}_{\mathrm{j}}$ ) (Moreno, 2001; Moreno, Barragán, Pineda, \& Pavón, 2011). La importancia ecológica de las especies se expresó mediante el Valor de Importancia Relativa [VIR $(\%)=1 / 3$ (Densidad Relativa + Área Basal Relativa + Frecuencia Relativa)]. Además se calculó, la densidad (número de individuos/hectárea, ind/ha), frecuencia y área basal, utilizando procedimientos estándar (Matteucci \& Colma, 1982; Mueller-Dombois \& Ellenberg, 1974). Se realizaron pruebas de medias ( $t$ Student) de las variables mencionadas, entre los grupos formados para reconocer las diferencias.

\section{RESULTADOS}

Composición florística: En la SBC de las tres áreas muestreadas se registró un total de 120 especies de plantas leñosas (incluidos todos, DAP $>2.5 \mathrm{~cm}>$ DAP), distribuidas en 80 géneros y 43 familias; de estas últimas, las más diversas fueron Fabaceae (34 spp.), Rubiaceae (nueve spp.), Burseraceae (ocho spp.) y Euphorbiaceae (ocho spp.) (Cuadro 2). A estas cuatro familias pertenece cerca de la mitad (49 \%) de las especies registradas; del resto, el $19 \%$ están representadas sólo por un taxón. El $30 \%$ de los géneros fueron representados por una sola especie, entre los más diversos estuvieron Bursera (siete spp.), Acacia (seis spp.), Lonchocarpus (cinco spp.) у Coccoloba (cuatro spp.). 
CUADRO 2

Número de géneros y especies, por familias registradas en tres áreas de SBC en el Istmo-Costa de Oaxaca

TABLE 2

Number of genera and species, by families registered in three areas of SBC in the Istmo-Costa of Oaxaca

\begin{tabular}{|c|c|c|c|}
\hline Familia & Géneros & $\begin{array}{l}\text { Especies con } \\
\mathrm{DAP} \leq 2.5 \mathrm{~cm}\end{array}$ & $\begin{array}{l}\text { Especies con } \\
\text { DAP } \geq 2.5 \mathrm{~cm}\end{array}$ \\
\hline Fabaceae & 17 & 34 & 23 \\
\hline Rubiaceae & 6 & 9 & 7 \\
\hline Burseraceae & 1 & 8 & 7 \\
\hline Euphorbiaceae & 3 & 7 & 4 \\
\hline Cactaceae & 5 & 5 & 2 \\
\hline Apocynaceae & 3 & 4 & 4 \\
\hline Polygonaceae & 1 & 4 & 4 \\
\hline Anacardiaceae & 3 & 3 & 3 \\
\hline Asteraceae & 3 & 3 & 1 \\
\hline Primulaceae & 1 & 3 & 2 \\
\hline Tiliaceae & 2 & 3 & 4 \\
\hline Boraginaceae & 1 & 2 & 2 \\
\hline Capparaceae & 2 & 2 & 2 \\
\hline Malvaceae & 2 & 2 & 1 \\
\hline Moraceae & 2 & 2 & 2 \\
\hline Rutaceae & 1 & 2 & 1 \\
\hline Annonaceae & 1 & 1 & 0 \\
\hline Bignoniaceae & 1 & 1 & 2 \\
\hline Bixaceae & 1 & 1 & 1 \\
\hline Bombacaceae & 1 & 1 & 1 \\
\hline Bromeliaceae & 1 & 1 & 0 \\
\hline Caricaceae & 1 & 1 & 1 \\
\hline Celastraceae & 1 & 1 & 1 \\
\hline Combretaceae & 1 & 1 & 1 \\
\hline Ebenaceae & 1 & 1 & 1 \\
\hline Erythroxylaceae & 1 & 1 & 1 \\
\hline Flacourtiaceae & 1 & 1 & 1 \\
\hline Hernandiaceae & 1 & 1 & 1 \\
\hline Lamiaceae & 1 & 1 & 0 \\
\hline Lauraceae & 1 & 1 & 1 \\
\hline Malphigiaceae & 1 & 1 & 1 \\
\hline Meliaceae & 1 & 1 & 1 \\
\hline Opiliaceae & 1 & 1 & 1 \\
\hline Phyllanthaceae & 1 & 1 & 1 \\
\hline Poaceae & 1 & 1 & 0 \\
\hline Resedaceae & 1 & 1 & 1 \\
\hline Rhamnaceae & 1 & 1 & 1 \\
\hline Sapindaceae & 1 & 1 & 1 \\
\hline Solanaceae & 1 & 1 & 0 \\
\hline Ulmaceae & 1 & 1 & 0 \\
\hline Urticaceae & 1 & 1 & 1 \\
\hline Verbenaceae & 1 & 1 & 0 \\
\hline Zygophyllaceae & 1 & 1 & 1 \\
\hline TOTALES & 80 & 120 & 90 \\
\hline
\end{tabular}

Curva de acumulación de especies. De acuerdo con el inventario florístico (con DAP $\geq$ $2.5 \mathrm{~cm}$ ) de la $\mathrm{SBC}$ en las tres áreas y representada con la curva de acumulación de especies (Fig. 2), considera un porcentaje cercano al de los estimadores Jackknife (80 \%) y ICE (85\%). Faltarían de 16 a 23 especies de leñosas por registrar para alcanzar el $100 \%$ del inventario y el nivel asintótico de la curva. En el caso particular de cada área, la representación fue menor, del $69 \%$ a $75 \%$ (Fig. 3).

Composición de leñosas con DAP $\geq 2.5$. En total se registraron 90 especies (inventariadas en cuadros de $100 \mathrm{~m}^{2}$ de las tres áreas), pertenecientes a 65 géneros y 36 familias; de las cuales Fabaceae presentó la mayor riqueza de géneros (14) y especies (24), seguida por Rubiaceae (seis géneros y siete spp.), Euphorbiaceae (tres géneros y cuatro spp.) y Apocynaceae (tres géneros y cuatro spp.); las Burseraceae y Polygonaceae solo registraron un género, con siete y cuatro especies respectivamente (Cuadro 2). Del total de las especies registradas, 85 son árboles, tres arbustos, un bejuco y una arborescente (de la familia Cactaceae).

Diversidad. La riqueza de especies y diversidad verdadera en las diferentes áreas fue: $\mathrm{ADUC}, \mathrm{S}=50,{ }^{\mathrm{q}} \mathrm{D}=27$; $\mathrm{ADVC}, \mathrm{S}=55$, ${ }^{\mathrm{q}} \mathrm{D}=30$; ANP, $\mathrm{S}=48,{ }^{\mathrm{q}} \mathrm{D}=27$. El área mejor conservada (ANP) tiene menor riqueza y la diversidad verdadera y se asemejan con la de ADUC. Las especies más abundantes en el ADUC fueron Acacia macracantha, Cnidoscolus megacanthus, Thouinia villosa y Piptadenia obliqua; en el ADVC, C. megacanthus, Acacia hayesii, Amphipterygium adstringens y Bucida wigginsiana; y en el ANP, Comocladia mollissima, Coccoloba sp., Tabernaemontana amygdalifolia y Luehea aff. speciosa (Cuadro 3). La diversidad beta (índice de Jaccard) entre el ANP y ADVC $\mathrm{I}_{\mathrm{j}}=0.22$, la del ANP y ADUC $\mathrm{I}_{\mathrm{j}}=0.18$ y entre ADUC y ADVC de $\mathrm{I}_{\mathrm{j}}=0.30$.

La similitud florística entre las 45 parcelas agrupa el área mejor conservada (ANP) y mezcla las correspondientes a el ADVC y el ADUC (Fig. 4), separando a dos grupos bien definidos, G1 y G2, con una disimilitud de 0.1 , que corresponde aproximadamente a un $90 \%$ 


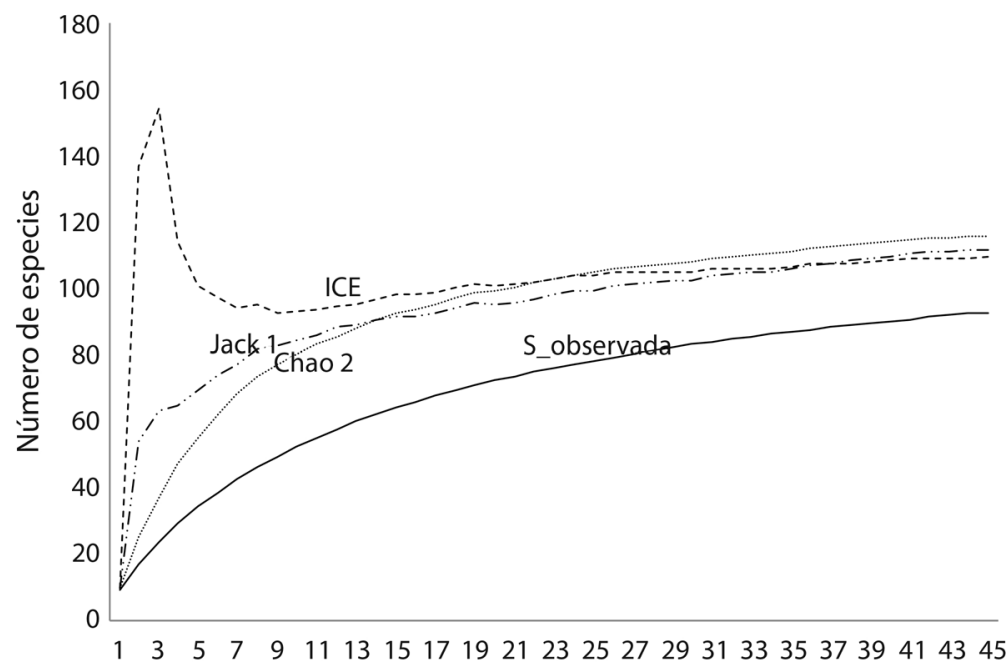

Fig. 2. Curvas de acumulación (observada con linea oscura) y estimada con líneas claras) de especies leñosas registradas en las áreas (ADUC, ADVC y ANP) de la SBC del Istmo-Costa, de Oaxaca, México.

Fig. 2. Accumulation curves (observed with dark line) and estimated with light lines) of woody species registered in the areas (ADUC, ADVC and ANP) of the SBC of Istmo-Costa, Oaxaca, Mexico.

de disimilitud entre el ANP (G1) y las ADVC y ADUC (G2). El G1 presenta 53 especies, de las cuales 21 (23\%) son exclusivas del grupo, entre ellas Ceiba aesculifolia, Comocladia mollissima, Luehea aff. speciosa y Swietenia humilis. Al G2 pertenecen 69 especies, 37 (41 $\%)$ de ellas exclusivas, como Acacia acatlensis, A. hayesii, A. macracantha y Cnidoscolus megacanthus (Cuadro 3). El $36 \%$ de las especies fueron comunes en los dos grupos (Fig. 4).

Diversidad entre grupos. La riqueza de especies en el G1 se ubicó en un intervalo de 2 a 18 , con promedio de 8 ; en el G2, el rango fue de 2 a 19, con una media de 10. Asimismo, los índices de Shannon, dominancia de Simpson y la diversidad verdadera de Jost para el G1 correspondieron a $\mathrm{H}^{\prime}=2.43, \mathrm{D}=0.21$ y ${ }^{\mathrm{q}} \mathrm{D}=$ 11 , menores que en el $\mathrm{G} 2, \mathrm{H}^{\prime}=2.76, \mathrm{D}=0.28$ y ${ }^{\mathrm{q}} \mathrm{D}=15$. El análisis de medias $\left(\mathrm{H}^{\prime}, \mathrm{t}=-1.920\right.$, $\mathrm{P}=0.120 ; \mathrm{D}, \mathrm{t}=0.053 ; \mathrm{P}=0.958$ y ${ }^{\mathrm{q}} \mathrm{D}, \mathrm{t}=0.941$, $\mathrm{P}=0.924)$ no mostró diferencias significativas de estos índices entre los dos grupos. La estimación de la diversidad beta fue de $\mathrm{I}_{\mathrm{j}}=0.27$, es decir que el porcentaje de especies compartidas se asemeja al obtenido entre pares de áreas.
La densidad de especies leñosas con DAP $\geq 2.5 \mathrm{~cm} \mathrm{del} \mathrm{G1} \mathrm{fue} \mathrm{de} 1829 \mathrm{ind} / \mathrm{ha}$, para el G2 de $2370 \mathrm{ind} / \mathrm{ha}$. El promedio de altura (6.8 m) y área basal $\left(19.7 \mathrm{~m}^{2} / \mathrm{ha}\right)$ en el G1 fueron menores que en el G2 (7.9 $\mathrm{m}$ y $\left.22.1 \mathrm{~m}^{2} / \mathrm{ha}\right)$. La prueba de comparación de medias $(\mathrm{t}=0.053$, $\mathrm{P}=0.958$ ) entre grupos para estas variables no mostraron diferencias significativas.

Las especies con mayor valor de importancia ecológica (VIR, dado por la relación entre área basal, densidad y frecuencia relativa) en los grupos formados difieren entre sí. En el G1 fueron Acacia cornigera, Coccoloba acapulcensis, Comocladia mollissima y Luehea aff. speciosa. En el G2 Acacia hayesii, Amphipterygium adstringens, Cnidoscolus megacanthus y Piptadenia obliqua (Cuadro 3).

En cuanto a los juveniles, se observaron diferencias significativas $(\mathrm{t}=3.870, \mathrm{P}=0.05)$ entre la densidad en el G1 (9 $583.3 \mathrm{ind} / \mathrm{ha})$ y la del G2 (11 587 ind/ha). En el G1, Acacia hayesii, Diospyros salicifolia y Piptadenia obliqua registraron el mayor número de individuos; mientras que en el G2 fueron Apoplanesia paniculata, D. salicifolia y Heliocarpus donnellsmithii (Cuadro 3). 

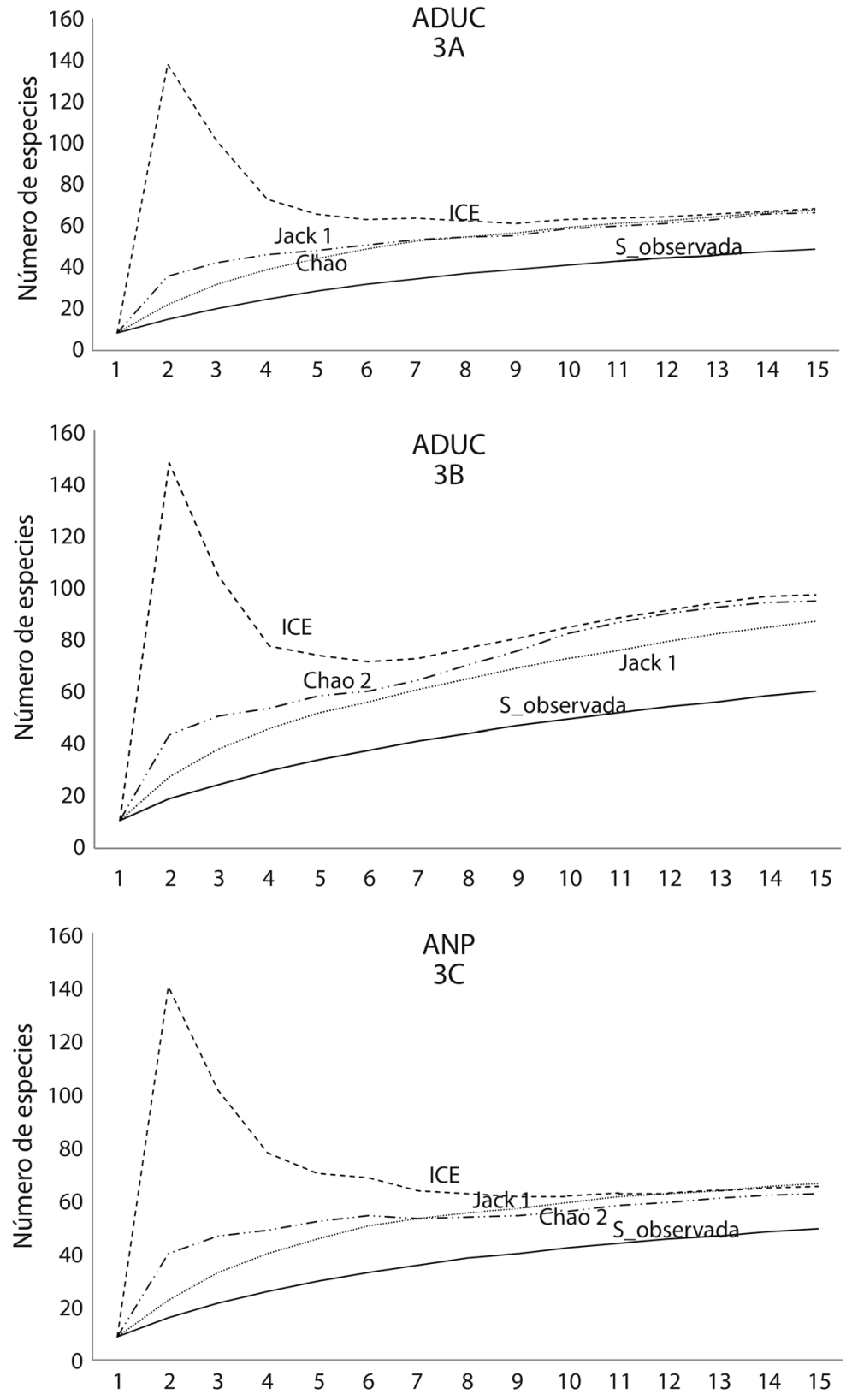

Fig. 3. Curvas de acumulación (observada con línea oscura y estimada líneas claras) de especies leñosas, según tipo de área ( $3 \mathrm{~A}=\mathrm{ADUC}, 3 \mathrm{~B}=\mathrm{ADVC}, 3 \mathrm{C}=\mathrm{ANP}$ ) en la $\mathrm{SBC}$ del Istmo-Costa, Oaxaca, México.

Fig. 3. Accumulation curves (observed with dark line and estimated light lines) of woody species, according to the type of area $(3 \mathrm{~A}=\mathrm{ADUC}, 3 \mathrm{~B}=\mathrm{ADVC}, 3 \mathrm{C}=\mathrm{ANP})$ at $\mathrm{SBC}$ Istmo-Costa, Oaxaca, Mexico. 
CUADRO 3

Valores estructurales, valor de importancia relativa (VIR) y forma biológica (FB) para las especies leñosas (DAP $\geq 2.5 \mathrm{~cm}$ ) registradas (G1=ANP Parque Nacional Huatulco y G2=ADVC y ADUC de San Pedro Huamelula) en 45 cuadros de muestreo en SBC del Istmo de Oaxaca

TABLE 3

Structural values, value of relative importance (VIR) and biological form (FB) for the woody species (DAP $\geq 2.5 \mathrm{~cm}$ ) registered (G1=ANP National park Huatulco and G2=ADVC and ADUC of San Pedro Huamelula) in 45 sampling in SBC of the Istmo of Oaxaca

\begin{tabular}{|c|c|c|c|c|c|c|c|c|c|c|}
\hline Especie & Grupo & FB & $\mathrm{N}$ & DR & $\mathrm{F}$ & FR & $\mathrm{ABR}$ & $\begin{array}{c}\text { Dominancia } \\
\left(\mathrm{m}^{2} / \mathrm{ha}\right)\end{array}$ & $\begin{array}{c}\text { Número de } \\
\text { juveniles }\end{array}$ & VIR \\
\hline & & & & & & & & & 115 & 100 \\
\hline Comocladia mollissima Kunth & G1 & A & 28 & 0.09 & 5 & 0.04 & 0.13 & 6.79 & 0 & 8.65 \\
\hline Luehea aff. speciosa Willd. & G1 & A & 12 & 0.04 & 2 & 0.01 & 0.18 & 20.99 & 1 & 7.66 \\
\hline Acacia cornigera (L.) Willd. & G1 & A & 23 & 0.07 & 9 & 0.07 & 0.06 & 3.98 & 3 & 6.78 \\
\hline Coccoloba acapulcensis Standl & G1 & A & 13 & 0.04 & 5 & 0.04 & 0.1 & 10.9 & 0 & 5.91 \\
\hline Heliocarpus donnellsmithii Rose & G1 & A & 12 & 0.04 & 10 & 0.07 & 0.04 & 4.54 & 8 & 4.98 \\
\hline Tabernaemontana amygdalifolia Seem. & G1 & $\mathrm{A}$ & 16 & 0.05 & 6 & 0.04 & 0.03 & 2.81 & 3 & 4.22 \\
\hline Bucida wigginsiana Miranda & G1 & A & 7 & 0.02 & 1 & 0.01 & 0.09 & 19.11 & 3 & 4.12 \\
\hline Cochlospermum vitifolium (Willd.) Spreng. & G1 & A & 8 & 0.03 & 4 & 0.03 & 0.06 & 10.64 & 1 & 3.81 \\
\hline Coccoloba sp. & G1 & A & 15 & 0.05 & 5 & 0.04 & 0.03 & 2.51 & 7 & 3.69 \\
\hline Bursera coyucensis Bullock & G1 & A & 10 & 0.03 & 4 & 0.03 & 0.03 & 4.88 & 0 & 3.18 \\
\hline Perrottetia sp. & G1 & A & 10 & 0.03 & 5 & 0.04 & 0.01 & 1.35 & 0 & 2.59 \\
\hline Apoplanesia paniculata C. Presl & G1 & A & 10 & 0.03 & 4 & 0.03 & 0.01 & 1.69 & 16 & 2.43 \\
\hline Maclura tinctoria (L.) D. Don ex Steud. & G1 & A & 6 & 0.02 & 4 & 0.03 & 0.02 & 4.51 & 0 & 2.24 \\
\hline Poeppigia procera C. Presl & G1 & A & 6 & 0.02 & 5 & 0.04 & 0.01 & 2.5 & 2 & 2.2 \\
\hline Casearia hintonii Lundell & G1 & A & 8 & 0.03 & 4 & 0.03 & 0.01 & 1.86 & 3 & 2.17 \\
\hline Acacia cochliacantha Humb. \& Bonpl. ex Willd. & G1 & A & 5 & 0.02 & 3 & 0.02 & 0.03 & 7.44 & 1 & 2.13 \\
\hline Jacquinia aurantiaca W.T. Aiton & G1 & A & 8 & 0.03 & 4 & 0.03 & 0.01 & 1.61 & 0 & 2.12 \\
\hline Swietenia humilis Zucc. & G1 & A & 5 & 0.02 & 5 & 0.04 & 0 & 0.75 & 1 & 1.83 \\
\hline Tabebuia rosea (Bertol.) DC. & G1 & A & 9 & 0.03 & 2 & 0.01 & 0.01 & 1.18 & 8 & 1.7 \\
\hline Coccoloba aff. hondurensis Lundell & G1 & A & 8 & 0.03 & 2 & 0.01 & 0.01 & 1.79 & 4 & 1.68 \\
\hline Jacaratia dolichaula (Donn. Sm.) Woodson & G1 & A & 5 & 0.02 & 2 & 0.01 & 0.02 & 4.68 & 0 & 1.57 \\
\hline Croton fantzianus F. Seym. & G1 & $\mathrm{AR}$ & 6 & 0.02 & 1 & 0.01 & 0.02 & 4.78 & 0 & 1.55 \\
\hline Lonchocarpus palmeri Rose & G1 & A & 6 & 0.02 & 3 & 0.02 & 0 & 0.91 & 3 & 1.5 \\
\hline Karwinskia humboldtiana (Schult.) Zucc. & G1 & A & 6 & 0.02 & 2 & 0.01 & 0.01 & 2.51 & 1 & 1.48 \\
\hline Bursera simaruba (L.) Sarg. & G1 & A & 4 & 0.01 & 3 & 0.02 & 0.01 & 2.66 & 0 & 1.4 \\
\hline Thevetia plumeriifolia Benth. & G1 & A & 4 & 0.01 & 3 & 0.02 & 0.01 & 2.13 & 0 & 1.35 \\
\hline Bursera silviae Rzed. \& Calderón & G1 & A & 4 & 0.01 & 3 & 0.02 & 0 & 1.62 & 0 & 1.3 \\
\hline Bunchosia aff. nitida (Jacq.) DC. & G1 & $\mathrm{AR}$ & 8 & 0.03 & 1 & 0.01 & 0.01 & 0.9 & 0 & 1.27 \\
\hline Bourreria rekoi Standl. & G1 & A & 4 & 0.01 & 3 & 0.02 & 0 & 0.69 & 0 & 1.22 \\
\hline Lonchocarpus longipedicellatus Pittier & G1 & A & 2 & 0.01 & 1 & 0.01 & 0.02 & 14.33 & 0 & 1.13 \\
\hline Ocotea $\mathrm{sp}$ & G1 & A & 4 & 0.01 & 1 & 0.01 & 0.01 & 1.79 & 2 & 0.84 \\
\hline Croton niveus Jacq. & G1 & $\mathrm{AR}$ & 3 & 0.01 & 2 & 0.01 & 0 & 0.26 & 1 & 0.82 \\
\hline Euphorbia schlechtendalii Boiss. & G1 & A & 3 & 0.01 & 2 & 0.01 & 0 & 0.23 & 0 & 0.82 \\
\hline Gliricidia sepium Kunth ex Steud. & G1 & A & 2 & 0.01 & 2 & 0.01 & 0 & 2.7 & 0 & 0.82 \\
\hline Haematoxylum brasiletto H. Karst. & G1 & A & 2 & 0.01 & 2 & 0.01 & 0 & 0.5 & 2 & 0.72 \\
\hline Diospyros salicifolia Humb. \& Bonpl. ex Willd. & G1 & A & 4 & 0.01 & 1 & 0.01 & 0 & 0.42 & 9 & 0.71 \\
\hline Thouinia villosa DC. & G1 & A & 3 & 0.01 & 1 & 0.01 & 0 & 0.66 & 5 & 0.61 \\
\hline Ceiba aesculifolia (Kunth) Britten \& Baker f. & G1 & A & 1 & 0 & 1 & 0.01 & 0.01 & 8.6 & 0 & 0.55 \\
\hline
\end{tabular}


CUADRO 3 (Continuación) / TABLE 3 (Continued)

\begin{tabular}{|c|c|c|c|c|c|c|c|c|c|c|}
\hline Especie & Grupo & FB & $\mathrm{N}$ & DR & $\mathrm{F}$ & FR & $\mathrm{ABR}$ & $\begin{array}{c}\text { Dominancia } \\
\left(\mathrm{m}^{2} / \mathrm{ha}\right)\end{array}$ & $\begin{array}{l}\text { Número de } \\
\text { juveniles }\end{array}$ & VIR \\
\hline Urera pacifica V.W. Steinm. & G1 & $\mathrm{AR}$ & 2 & 0.01 & 1 & 0.01 & 0 & 1.16 & 0 & 0.51 \\
\hline Plumeria rubra L. & G1 & A & 2 & 0.01 & 1 & 0.01 & 0 & 0.96 & 2 & 0.5 \\
\hline Leucaena leucocephala (Lam.) de Wit & G1 & A & 2 & 0.01 & 1 & 0.01 & 0 & 0.78 & 4 & 0.49 \\
\hline Lasianthaea aff. fruticosa (L.) K.M. Becker & G1 & $\mathrm{AR}$ & 2 & 0.01 & 1 & 0.01 & 0 & 0.44 & 5 & 0.48 \\
\hline Spondias $\mathrm{sp}$ & G1 & A & 2 & 0.01 & 1 & 0.01 & 0 & 0.23 & 0 & 0.47 \\
\hline Coccoloba venosa $\mathrm{L}$. & G1 & A & 2 & 0.01 & 1 & 0.01 & 0 & 0.15 & 0 & 0.46 \\
\hline Gyrocarpus jatrophifolius Domin & G1 & A & 1 & 0 & 1 & 0.01 & 0 & 3.56 & 2 & 0.43 \\
\hline Stenocereus sp & G1 & $\mathrm{ARB}$ & 1 & 0 & 1 & 0.01 & 0 & 2.46 & 3 & 0.41 \\
\hline Crataeva tapia $\mathrm{L}$. & G1 & A & 1 & 0 & 1 & 0.01 & 0 & 1.15 & 5 & 0.38 \\
\hline Agonandra obtusifolia Standl. & G1 & A & 1 & 0 & 1 & 0.01 & 0 & 0.52 & 0 & 0.36 \\
\hline Amphipterygium adstringens (Schltdl.) Standl. & G1 & A & 1 & 0 & 1 & 0.01 & 0 & 0.32 & 1 & 0.36 \\
\hline Forchhammeria pallida Liebm. & G1 & A & 1 & 0 & 1 & 0.01 & 0 & 0.11 & 0 & 0.35 \\
\hline Heliocarpus aff. occidentalis Rose & G1 & A & 1 & 0 & 1 & 0.01 & 0 & 0.11 & 0 & 0.35 \\
\hline Jacquinia pringlei Bartlett & G1 & A & 1 & 0 & 1 & 0.01 & 0 & 0.08 & 4 & 0.35 \\
\hline Lysiloma microphyllum Benth. & G1 & A & 1 & 0 & 1 & 0.01 & 0 & 0.05 & 5 & 0.35 \\
\hline Total & G1 & & 311 & 1 & 138 & 1 & 1 & 174.25 & 146 & 100 \\
\hline Cnidoscolus megacanthus Breckon & G2 & A & 71 & 0.11 & 18 & 0.07 & 0.258 & 35.49 & 2 & 14.55 \\
\hline Piptadenia obliqua (Pers.) J.F. Macbr. & G2 & A & 44 & 0.07 & 14 & 0.05 & 0.18 & 39.95 & 12 & 10.04 \\
\hline Acacia hayesii Benth. & G2 & $\mathrm{A}$ & 43 & 0.07 & 12 & 0.04 & 0.096 & 21.83 & 15 & 6.94 \\
\hline Amphipterygium adstringens (Schltdl.) Standl. & G2 & A & 34 & 0.05 & 13 & 0.05 & 0.103 & 29.59 & 1 & 6.82 \\
\hline Stenocereus sp. & G2 & $\mathrm{ARB}$ & 19 & 0.03 & 12 & 0.04 & 0.068 & 34.96 & 3 & 4.75 \\
\hline Acacia macracantha Humb. \& Bonpl. ex Willd. & G2 & A & 36 & 0.06 & 5 & 0.02 & 0.059 & 16.06 & 1 & 4.47 \\
\hline Lonchocarpus lineatus Pittier & G2 & A & 23 & 0.04 & 11 & 0.04 & 0.014 & 5.84 & 8 & 3.02 \\
\hline Diospyros salicifolia Humb. \& Bonpl. ex Willd. & G2 & A & 24 & 0.04 & 11 & 0.04 & 0.011 & 4.64 & 9 & 2.99 \\
\hline Thouinia villosa DC. & G2 & A & 30 & 0.05 & 4 & 0.01 & 0.028 & 9.05 & 5 & 2.99 \\
\hline Bursera excelsa (Kunth) Engl. & G2 & A & 17 & 0.03 & 10 & 0.04 & 0.02 & 11.32 & 0 & 2.78 \\
\hline Tabernaemontana donnell-smithii Rose. & G2 & A & 18 & 0.03 & 9 & 0.03 & 0.014 & 7.79 & 5 & 2.53 \\
\hline Apoplanesia paniculata C. Presl & G2 & A & 18 & 0.03 & 6 & 0.02 & 0.019 & 10.33 & 7 & 2.32 \\
\hline Cochlospermum vitifolium (Willd.) Spreng. & G2 & A & 6 & 0.01 & 6 & 0.02 & 0.029 & 47.16 & 1 & 2.02 \\
\hline Randia aff. armata (Sw.) DC. & G2 & A & 19 & 0.03 & 4 & 0.01 & 0.005 & 2.55 & 7 & 1.65 \\
\hline Bucida wigginsiana Miranda & G2 & A & 14 & 0.02 & 2 & 0.01 & 0.018 & 12.27 & 3 & 1.56 \\
\hline Gyrocarpus jatrophifolius Domin & G2 & A & 8 & 0.01 & 6 & 0.02 & 0.01 & 11.86 & 2 & 1.48 \\
\hline Jacquinia pringlei Bartlett & G2 & A & 14 & 0.02 & 5 & 0.02 & 0.004 & 2.66 & 3 & 1.47 \\
\hline Lasianthaea aff. fruticosa (L.) K.M. Becker & G2 & $\mathrm{AR}$ & 13 & 0.02 & 6 & 0.02 & 0.001 & 1.07 & 5 & 1.47 \\
\hline Crataeva tapia $\mathrm{L}$. & G2 & A & 12 & 0.02 & 3 & 0.01 & 0.011 & 9.3 & 3 & 1.38 \\
\hline Lonchocarpus sp. & G2 & A & 9 & 0.01 & 6 & 0.02 & 0.008 & 6.54 & 0 & 1.24 \\
\hline Maclura tinctoria (L.) D. Don ex Steud. & G2 & A & 9 & 0.01 & 4 & 0.01 & 0.008 & 8.67 & 0 & 1.23 \\
\hline Lysiloma microphyllum Benth. & G2 & A & 6 & 0.01 & 6 & 0.02 & 0.003 & 4.5 & 3 & 1.15 \\
\hline Lonchocarpus palmeri Rose & G2 & A & 10 & 0.02 & 7 & 0.03 & 0.002 & 2.02 & 2 & 1.1 \\
\hline Heliocarpus donnellsmithii Rose & G2 & A & 7 & 0.01 & 5 & 0.02 & 0.003 & 4.64 & 6 & 1.09 \\
\hline Palicourea sp. & G2 & A & 7 & 0.01 & 4 & 0.01 & 0.001 & 1.74 & 2 & 0.9 \\
\hline Mimosa sp. & G2 & A & 7 & 0.01 & 3 & 0.01 & 0.005 & 6.34 & 0 & 0.89 \\
\hline Genipa americana $\mathrm{L}$. & G2 & A & 4 & 0.01 & 5 & 0.02 & 0.002 & 4.28 & 1 & 0.88 \\
\hline Arrabidaea sp. & G2 & B & 5 & 0.01 & 4 & 0.01 & 0.001 & 1.39 & 3 & 0.78 \\
\hline Guaiacum coulteri A. Gray & G2 & $\mathrm{A}$ & 8 & 0.01 & 2 & 0.01 & 0.003 & 3.92 & 0 & 0.77 \\
\hline Zanthoxylum aguilarii Standl. \& Steyerm. & G2 & $\mathrm{A}$ & 7 & 0.01 & 3 & 0.01 & 0.001 & 0.98 & 0 & 0.76 \\
\hline Forchhammeria pallida Liebm. & G2 & A & 4 & 0.01 & 4 & 0.01 & 0.001 & 3.19 & 0 & 0.75 \\
\hline
\end{tabular}


CUADRO 3 (Continuación) / TABLE 3 (Continued)

\begin{tabular}{|c|c|c|c|c|c|c|c|c|c|c|}
\hline Especie & Grupo & FB & $\mathrm{N}$ & DR & $\mathrm{F}$ & FR & $\mathrm{ABR}$ & $\begin{array}{c}\text { Dominancia } \\
\left(\mathrm{m}^{2} / \mathrm{ha}\right)\end{array}$ & $\begin{array}{l}\text { Número de } \\
\text { juveniles }\end{array}$ & VIR \\
\hline Piscidia carthagenensis Jacq. & G2 & A & 4 & 0.01 & 4 & 0.01 & 0.001 & 1.4 & 0 & 0.72 \\
\hline Erythroxylum mexicanum Kunth & $\mathrm{G} 2$ & A & 6 & 0.01 & 3 & 0.01 & 0.001 & 1 & 0 & 0.7 \\
\hline Euphorbia schlechtendalii Boiss. & $\mathrm{G} 2$ & A & 5 & 0.01 & 3 & 0.01 & 0 & 0.63 & 4 & 0.64 \\
\hline Chomelia sp. & $\mathrm{G} 2$ & A & 7 & 0.01 & 2 & 0.01 & 0.001 & 0.95 & 0 & 0.63 \\
\hline Bursera laurihuertae Rzed. \& Calderón & $\mathrm{G} 2$ & A & 4 & 0.01 & 3 & 0.01 & 0.001 & 1.52 & 0 & 0.6 \\
\hline Acacia acatlensis Benth. & $\mathrm{G} 2$ & A & 3 & 0 & 3 & 0.01 & 0.001 & 2.75 & 0 & 0.55 \\
\hline Diphysa sp. 1 & $\mathrm{G} 2$ & A & 3 & 0 & 3 & 0.01 & 0.001 & 2.25 & 0 & 0.55 \\
\hline Psychotria microdon (DC.) Urb. & $\mathrm{G} 2$ & A & 4 & 0.01 & 2 & 0.01 & 0.001 & 1.87 & 0 & 0.48 \\
\hline Coccoloba acapulcensis Standl & $\mathrm{G} 2$ & A & 4 & 0.01 & 2 & 0.01 & 0 & 0.73 & 5 & 0.47 \\
\hline Quadrella incana (Kunth) Iltis \& Cornejo & $\mathrm{G} 2$ & A & 4 & 0.01 & 2 & 0.01 & 0 & 0.89 & 0 & 0.47 \\
\hline Acacia cornigera (L.) Willd. & G2 & A & 3 & 0 & 2 & 0.01 & 0 & 0.78 & 0 & 0.41 \\
\hline Bursera simaruba (L.) Sarg. & $\mathrm{G} 2$ & A & 2 & 0 & 2 & 0.01 & 0.002 & 8.06 & 4 & 0.41 \\
\hline Leucaena leucocephala (Lam.) de Wit & G2 & A & 3 & 0 & 2 & 0.01 & 0 & 0.32 & 3 & 0.41 \\
\hline Mimosa goldmanii B.L. Rob. & $\mathrm{G} 2$ & A & 5 & 0.01 & 1 & 0 & 0 & 0.63 & 0 & 0.39 \\
\hline Cordia elaeagnoides DC. & $\mathrm{G} 2$ & A & 4 & 0.01 & 1 & 0 & 0.001 & 3.6 & 0 & 0.38 \\
\hline Acacia cochliacantha Humb. \& Bonpl. ex Willd. & $\mathrm{G} 2$ & A & 2 & 0 & 2 & 0.01 & 0 & 0.91 & 1 & 0.36 \\
\hline Bursera silviae Rzed. \& Calderón & G2 & A & 2 & 0 & 2 & 0.01 & 0 & 0.15 & 0 & 0.35 \\
\hline Plumeria rubra $\mathrm{L}$. & $\mathrm{G} 2$ & A & 3 & 0 & 1 & 0 & 0.001 & 4.11 & 2 & 0.32 \\
\hline Bunchosia aff. nitida (Jacq.) DC. & G2 & $\mathrm{AR}$ & 2 & 0 & 1 & 0 & 0 & 0.22 & 0 & 0.23 \\
\hline Diphysa sp. & G2 & A & 2 & 0 & 1 & 0 & 0 & 0.26 & 9 & 0.23 \\
\hline Lonchocarpus constrictus Pittier & G2 & A & 2 & 0 & 1 & 0 & 0 & 0.92 & 0 & 0.23 \\
\hline Psychotria sp. & $\mathrm{G} 2$ & A & 2 & 0 & 1 & 0 & 0 & 0.22 & 0 & 0.23 \\
\hline Spondias sp. & G2 & A & 2 & 0 & 1 & 0 & 0 & 0.99 & 0 & 0.23 \\
\hline Tabernaemontana amygdalifolia Seem. & $\mathrm{G} 2$ & A & 2 & 0 & 1 & 0 & 0 & 0.14 & 2 & 0.23 \\
\hline Pereskia lychnidiflora DC. & $\mathrm{G} 2$ & A & 1 & 0 & 1 & 0 & 0 & 4.6 & 1 & 0.19 \\
\hline Bursera graveolens (Kunth) Triana \& Planch. & $\mathrm{G} 2$ & A & 1 & 0 & 1 & 0 & 0 & 0.07 & 0 & 0.18 \\
\hline Bursera schlechtendalii Engl. & $\mathrm{G} 2$ & A & 1 & 0 & 1 & 0 & 0 & 0.05 & 0 & 0.18 \\
\hline Caesalpinia sp. & $\mathrm{G} 2$ & A & 1 & 0 & 1 & 0 & 0 & 0.31 & 0 & 0.18 \\
\hline Casearia hintonii Lundell & $\mathrm{G} 2$ & A & 1 & 0 & 1 & 0 & 0 & 0.14 & 0 & 0.18 \\
\hline Cordia sp. & $\mathrm{G} 2$ & A & 1 & 0 & 1 & 0 & 0 & 0.08 & 0 & 0.18 \\
\hline Ficus cotinifolia Kunth & $\mathrm{G} 2$ & A & 1 & 0 & 1 & 0 & 0 & 0.44 & 0 & 0.18 \\
\hline Gliricidia sepium Kunth ex Steud. & $\mathrm{G} 2$ & A & 1 & 0 & 1 & 0 & 0 & 0.12 & 1 & 0.18 \\
\hline Haematoxylum brasiletto H. Karst. & $\mathrm{G} 2$ & A & 1 & 0 & 1 & 0 & 0 & 0.09 & 0 & 0.18 \\
\hline Lonchocarpus longipedicellatus Pittier & G2 & A & 1 & 0 & 1 & 0 & 0 & 0.33 & 0 & 0.18 \\
\hline Lysiloma sp. & $\mathrm{G} 2$ & A & 1 & 0 & 1 & 0 & 0 & 1.31 & 0 & 0.18 \\
\hline Phyllanthus sp. & $\mathrm{G} 2$ & A & 1 & 0 & 1 & 0 & 0 & 0.61 & 0 & 0.18 \\
\hline Tabebuia rosea (Bertol.) DC. & $\mathrm{G} 2$ & A & 1 & 0 & 1 & 0 & 0 & 0.87 & 4 & 0.18 \\
\hline Triumfetta aff. grandiflora Vahl & G2 & $\mathrm{A}$ & 1 & 0 & 1 & 0 & 0 & 0.08 & 1 & 0.18 \\
\hline Total & G2 & & 640 & 1 & 270 & 1 & 1 & 406.33 & Total & G2 \\
\hline
\end{tabular}

$\mathrm{N}=$ número de individuos; $\mathrm{DR}=$ densidad relativa; $\mathrm{F}=$ frecuencia; $\mathrm{FR}=$ frecuencia relativa; $\mathrm{ABR}=$ área basal relativa. $\mathrm{A}=$ árbol, $\mathrm{AR}=$ arbusto, $\mathrm{B}=$ bejuco, $\mathrm{ARB}=$ arborescente. 


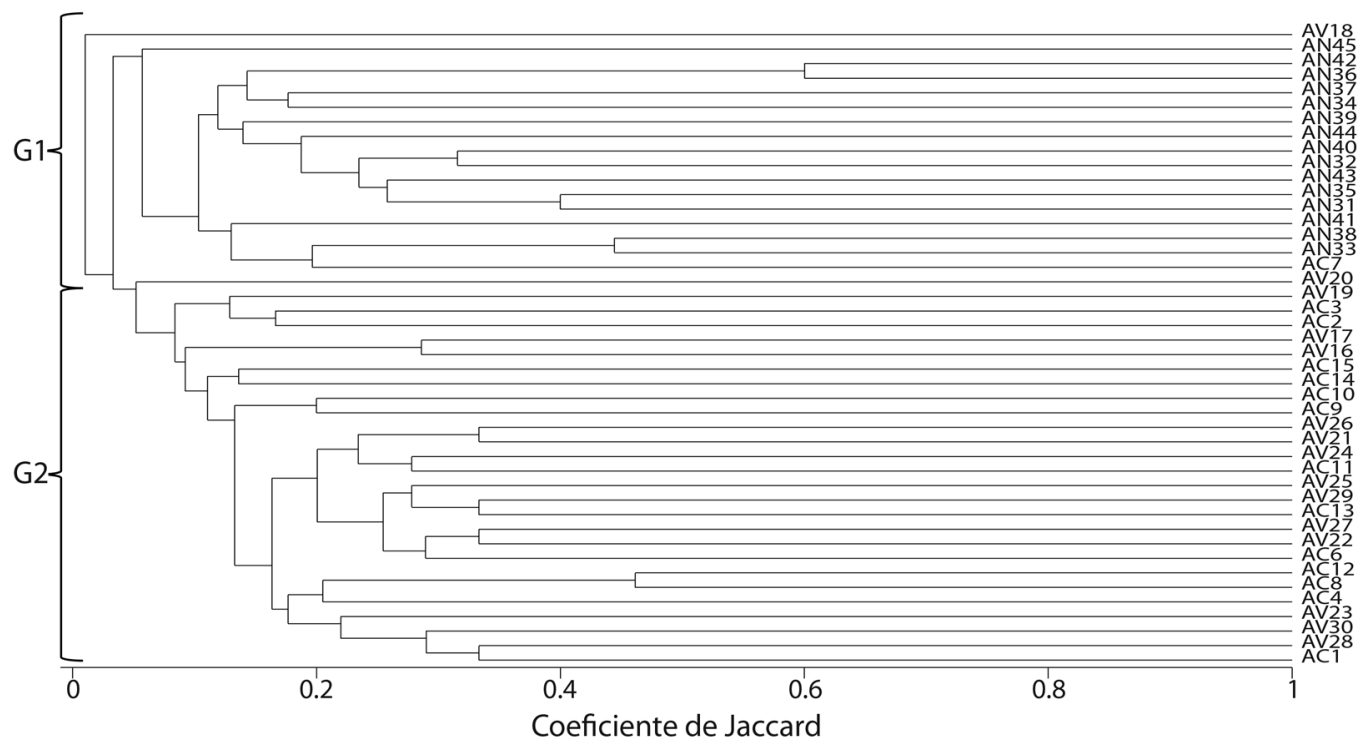

Fig. 4. Dendrograma de similitud de especies leñosas registradas en la SBC, por unidades de muestreo del ANP Parque Nacional Huatulco (AN) Grupo 1, ADVC (AV) y ADUC (AC) Grupo 2, de San Pedro Huamelula, basado en el coeficiente de Jaccard.

Fig. 4. Dendrogram of similarity of woody species recorded in the SBC, by ANP sampling units National Park Huatulco (AN) Group 1, ADVC (AV) and ADUC (AC) Group 2, of San Pedro Huamelula, based on the coefficient of Jaccard.

Endemismo. Se recolectaron cuatro especies endémicas del estado de Oaxaca Bourreria rekoi, Bursera laurihuertae, Bursera silviae y Lasianthaea fruticosa (Mendoza \& Meave, 2011). Las especies Pereskia lychnidiflora y Swietenia humilis se incluyen en la lista de CITES en el apéndice II; además, la última está categorizada como vulnerable en la relación de IUCN (CONABIO, 2012).

\section{DISCUSIÓN}

La SBC de las tres áreas, en la región Istmo-Costa, mantiene valores de riqueza y diversidad de especies de leñosas dentro del intervalo reportado para este tipo de vegetación en México (34 a 111 sp.) (Lott, Bullock, \& Solis-Magallanes, 1987; Trejo \& Dirzo, 2002; Durán, Meave, Lott, \& Segura, 2006; Hernández-Ramírez \& García Méndez, 2015) y otras regiones neotropicales (45 a $75 \mathrm{sp}$.) (Gentry, 1995; Murphy \& Lugo, 1995; Gillespie, Grijalva, \& Farris, 2000)genera (69).
Los valores del índice de diversidad de Shannon en las tres áreas muestreadas coinciden con lo estimado para otros bosques secos de México $\left(H^{\prime}=2.84\right.$ a 4.17) (Mizrahi, Ramos, \& Jiménez-Osornio, 1997; Trejo y Dirzo, 2002; Gallardo-Cruz et al., 2005; Durán et al., 2006; Williams-Linera \& Lorea, 2009)Yucatan, Mexico in 12- and 26-year-old secondary forests. Species richness and diversity were greater as the stand grew older. Even though $73 \%$ of species have the capacity to regenerate from coppiced shoots, only between 30 and $50 \%$ of the individuals actually regenerate from coppiced shoots in both communities, respectively. According to the Sorensen similarity index both stands are similar in composition (73\%).

En los grupos formados por las especies leñosas, G1 y G2, la riqueza y diversidad alfa no presentaron diferencias significativas, posiblemente debido a la alta diversidad local que este ecosistema (SBC) presenta (Durán et al., 2006; Gallardo-Cruz et al., 2005; Trejo, 2005), ya que metodológicamente se cuidó de 
que fueran ambientalmente lo más similares posible. En este mismo sentido se explicarían los resultados de la diversidad beta, tanto en áreas como en grupos, ya que los valores bajos de similitud indican un recambio alto de especies. La variación que muestra la SBC se puede deber tanto a las variaciones ambientales que prevalecen en cada uno de los sitios donde se establece, a lo que se suman las modificaciones que sufre este ecosistema por la intervención de las poblaciones humanas (Trejo \& Dirzo, 2002).

Durán et al. (2006) explican que en Chamela (Jalisco, México) las curvas de acumulación de especies, si bien indican la representatividad del muestreo, no se estabilizan, por lo que existe la posibilidad de que en distintas muestras de los mismos sitios el número de especies se mantenga, aunque su composición probablemente sería diferente. En este estudio, las curvas de acumulación (que mostraron una representatividad del $80 \%$ ) tampoco alcanzaron el nivel asintótico. Estos resultados indican que tanto el área protegida (como el Parque Nacional Huatulco) como la manejada por las poblaciones locales (ADUC) son de suma importancia para conservar la diversidad vegetal de la SBC; sin embargo, es necesario continuar su monitoreo para reconocer patrones de diversidad más propios del ecosistema; con ello también se esperaría que al aumentar el tiempo de restricción del ADVC se lograra diferenciar su composición de las parcelas del ADUC.

La variación en la estructura vegetal de las selvas bajas caducifolias se ha documentado a diferentes escalas, vinculándola con factores ambientales y de manejo (Trejo \& Dirzo, 2002; Durán et al., 2006; Banda et al., 2016). En Chamela, Durán et al. (2006) determinaron que la presencia de especies exclusivas de algunos sitios estaba relacionada con la litología superficial, en particular con la presencia de granito. En este trabajo, la composición de especies en los grupos G1 y G2, también observó exclusividad de algunas, lo que puede explicarse por las variaciones presentes en el paisaje; ya que las áreas más cercanas entre sí
(ADUC y ADVC) formaron uno de los grupos, separando las parecerlas del ANP. Sin embargo, algunas especies las reportan para diferentes etapas sucesionales (vegetación secundaria) (Pennington \& Sarukhán, 2005; Rocha, 2010population genetic structure and gene flow of the American crocodile (Crocodylus acutus; Jiménez-Ramírez \& Martínez-Gordillo, 2004), esto sugiere que la variación también está relacionada con la historia de uso o de perturbación natural (huracanes, incendios, etc.); la región Istmo-Costa de Oaxaca es una zona expuesta a huracanes con regular frecuencia (CONANP, 2003). Esto se corrobora con lo obtenido en el G2, donde el VIR fue mayor para Cnidoscolus megacanthus "palo tierno", Acacia hayesii "palo de cucharita" y Piptadenia obliqua "palo de concha", clasificadas como pioneras en el proceso de sucesión de la selva (Pennington \& Sarukhán, 2005; Rocha, 2010population genetic structure and gene flow of the American crocodile (Crocodylus acutus; Jiménez-Ramírez \& Martínez-Gordillo, 2004). En el ANP las especies con VIR alto también se consideran secundarias, pero de etapas más avanzadas de la sucesión, como Luehea aff. speciosa y Comocladia mollissima (Pennington \& Sarukhán, 2005; Rocha, 2010)population genetic structure and gene flow of the American crocodile (Crocodylus acutus. La composición de especies en los grupos formados, apoya lo señalado por otros autores (Murphy \& Lugo, 1986) sobre el proceso de recuperación en estos ecosistemas, considerado como lento; según Osorio (2012), se requiere de entre 60 y 80 años para una recuperación completa de la selva después de un disturbio.

Leiva, Rocha, Mata y Gutiérrez-Soto (2009) señalan que en el bosque tropical seco en áreas de Costa Rica, la densidad de individuos, el área basal por hectárea y la altura dominante aumentan con la edad del bosque. En este trabajo no se presentan diferencias significativas entre los grupos, pero el área basal registrada del G1 $\left(19.7 \mathrm{~m}^{2} / \mathrm{ha}\right)$ y la del G2 $\left(22.1 \mathrm{~m}^{2} / \mathrm{ha}\right)$ rebasan el límite inferior del intervalo global $\left(17-40 \mathrm{~m}^{2} / \mathrm{ha}\right)$ propuesto por Murphy y Lugo (1995) y es cercano a lo 
obtenido por Lott et al. (1987) $\left(24 \mathrm{~m}^{2} / \mathrm{ha}\right)$ para la SBC de Chamela.

La repoblación estimada por la densidad de los juveniles, en los grupos G1 (9 583.3 $\mathrm{ind} / \mathrm{ha}$ ) y G2 (1 $1587.3 \mathrm{ind} / \mathrm{ha})$ fue menor a la registrada por Gallardo-Cruz et al. (2005), de $19017.7 \mathrm{ind} / \mathrm{ha}$. Las diferencias podrían estar relacionadas con variaciones en el diseño de muestreo; en el presente trabajo se contabilizaron todos los individuos de especies leñosas a partir de $10 \mathrm{~cm}$ de altura y DAP $\geq 2.5 \mathrm{~cm}$, por su parte, los autores citados consideraron $\operatorname{los} \mathrm{DAP} \geq 1 \mathrm{~cm}$, con altura $\geq 30 \mathrm{~cm}$ (con esto, incluyeron leñosas y herbáceas).

La semejanza en la riqueza de especies y diversidad entre las áreas (ANP, ADVC y ADUC), así como en los grupos formados (G1 y G2) coincide con estudios que comparan zonas con y sin restricción de uso (Shackleton, 2000; Pérez et al., 2006; Neelo, Teketay, Kashe, \& Masamba, 2015; Abdallah, Belgacem, \& Chaieb, 2016). Las ADUC, ADVC (que han sido manejadas por las comunidades) y el ANP albergan parte de la diversidad de la SBC de la región. Sin embargo, la composición de especies sugiere que el ANP se ubica en una etapa avanzada de sucesión que las manejadas por las poblaciones locales, por lo que su condición de área protegida la incluye en un proceso de recuperación que a largo plazo la colocaría como área de vegetación primaria. Por otro lado, se considera que las ADVC y ADUC no están siendo totalmente devastadas. Sin embargo, se llega a advertir que la creciente presión por el uso de los recursos las coloca en estado de vulnerabilidad que puede transformarlas dramáticamente (Trejo \& Dirzo, 2002).

En conjunto, el ANP y las manejadas por comunidades locales de la región Istmo-Costa mantienen parte de la diversidad vegetal de la SBC, por lo que es imprescindible plantear políticas que incluyan los tres tipos de área, no solo en la elaboración y aplicación de planes de manejo y conservación restringida, pues la dinámica cotidiana de la gente, hasta el momento, favorece la estructura y diversidad de muchas de la selva.

\section{AGRADECIMIENTOS}

Agradecemos a los pobladores de San Pedro Huamelula, Playa Grande y El Morro Ayuta por el apoyo brindado para la realización de este trabajo; así como al personal de la Comisión de Áreas Naturales Protegidas de las regiones Istmo-Costa por las facilidades brindadas durante los muestreos y el acercamiento con los pobladores de los núcleos agrarios. La primera autora agradece al CONACYT la beca otorgada No. 100079.

\section{RESUMEN}

La Selva Baja Caducifolia (SBC) es de gran importancia por su biodiversidad. En el Istmo-Costa de Oaxaca, México, se encuentran áreas con SBC y distinta categoría de protección (legislación mexicana): (1) Área Natural Protegida (ANP), (2) Área Destinada Voluntariamente para la Conservación (ADVC) y (3) Área de Uso Común (ADUC). En cada una de ellas se analizó la estructura de vegetación leñosa, plantas con DAP $\geq 2.5 \mathrm{~cm}$, muestreando 15 parcelas de $100 \mathrm{~m}^{2}$. La riqueza observada fue de 90 especies incluidas en 36 familias y corresponde al $80 \%$ (Jackknife) y $85 \%$ (ICE) de la esperada. El análisis de agrupamiento identificó dos grupos con $90 \%$ de disimilitud: el primero (G1) incluyó a todas las parcelas muestreadas del ANP; y el segundo (G2) aglomeró las del ADVC y ADUC. Los índices de Shannon, dominancia de Simpson y diversidad verdadera de Jost para el $\mathrm{G} 1$ fueron $\mathrm{H}^{\prime}=2.43, \mathrm{D}=0.21$ y ${ }^{\mathrm{q}} \mathrm{D}=11$ y para el $\mathrm{G} 2, \mathrm{H}^{\prime}=2.76, \mathrm{D}=0.28$ y ${ }^{\mathrm{q}} \mathrm{D}=15$, sin diferencias entre grupos $(\mathrm{P}<0.05)$. Las especies con Valor de Importancia Relativa más elevado, en ambos grupos, corresponden a vegetación secundaria. La densidad de especies del G1 fue de 1829 ind/ha y en el G2 de 2370 ind/ha. Los promedios de altura $(6.8 \mathrm{~m}) \mathrm{y}$ área basal (19.7 $\left.\mathrm{m}^{2} / \mathrm{ha}\right)$ del $\mathrm{G} 1$ no variaron significativamente $(\mathrm{P}<0.05)$ con los del G2 (7.9 m y $\left.22.1 \mathrm{~m}^{2} / \mathrm{ha}\right)$. Las ADVC y ADUC, semejantes en composición y estructura, difieren del ANP. Se sugiere seguir monitoreando la SBC.

Palabras clave: Área Natural Protegida, áreas de uso común, áreas destinadas voluntariamente a la conservación, bosque tropical seco, diversidad de especies, similitud florística.

\section{REFERENCIAS}

Abdallah, F., Belgacem, A. O., \& Chaieb, M. (2016). Effects of ten years enclosure on vegetation characteristics of a national park in South Tunisia. Acta Botanica Gallica, 158, 161-168. 
Aguilar-Martínez, C., \& Arriaga, L. (2000). Deforestación y fragmentación de ecosistemas ¿Qué tan grave es el problema en México?. Biodiversitas, 30, 7-11.

Banda-R, K., Delgado-Salinas, A., Dexter K. G., LinaresPalomino, R., Oliveira-Filho, A., Prado, D.,... \& Weintritt, J. (2016). Plant diversity patterns in neotropical dry forests and their conservation implications. Science, 353(6303), 1383-1387.

Barajas-Morales, J., \& Pérez-Jiménez, L. A. P. (1990). Manual de identificación de árboles de selva baja mediante corteza (Vol. 6). México: UNAM, Instituto de Biología.

Bertzky, B. Corrigan, C., Kemsey, J., Kenney, S. Ravilious, C., Besançon, C., \& Burgess, N. (2012). Protected Planet Report 2012: Tracking progress towards global targets for protected areas. Gland, Switzerland: IUCN, UNEP-WCMC.

Chave, J. (2005). Medición de la altura del árbol, para árboles tropicales. Manual de campo. Toulouse, France: Université Paul Sabatier.

Chazdon, R. L., Colwell, R. K., Denslow, J. S., \& Guariguanta, M. R. (1998). Statistical methods for estimating species richness of woody regeneration in primary and secondary rain forest of Northeastern Costa Rica. Man and the Biosphere Series, 20, 286-309.

Chuvieco, E. (2000). Fundamentos de Teledetección. Madrid, España: RIALP.

Colwell, R. K. (2013). EstimateS v. 9.1.0: Statistical estimation of species richness and shared species from samples. Recuperado de http://viceroy.eeb.uconn. edu/estimates/

CONANP. (2003). Programa de Manejo: Parque Nacional Huatulco. México, D.F: Comisión Nacional de Áreas Naturales Protegidas.

CONANP. (2015). Áreas Destinadas Voluntariamente para la Conservación. Recuperado de http://www.conanp. gob.mx/que_hacemos/areas_certi.php

Cortés-Marcial, M., \& Briones-Salas, M. (2014). Diversidad, abundancia relativa y patrones de actividad de mamíferos medianos y grandes en una selva seca del Istmo de Tehuantepec, Oaxaca, México. Revista de Biología Tropical, 62(4), 1433-1448.

Dirzo, R. (1990). La Biodiversidad como crisis ecológica actual ¿Qué sabemos?. Ciencias, 4, 48-55.

Durán, E., Meave, J. A., Lott, E. J., \& Segura, G. (2006). Structure and tree diversity patterns at the landscape level in a Mexican tropical deciduous forest. Boletín de La Sociedad Botánica de México, 79, 43-63.

Gallardo-Cruz, J.A., Meave, J. A., \& Pérez-García, E.A. (2005). Estructura, composición y diversidad de la selva baja caducifolia del Cerro Verde, Nizanda (Oaxaca), México. Boletín de La Sociedad Botánica de México, 76, 19-35.

Gallardo-Cruz, J.A., Pérez-García, E.A., \& Meave, J.A. (2009). Diversity and vegetation structure as influenced by slope aspect and altitude in a seasonally dry tropical landscape. Landscape Ecology, 24, 473-482.

Gallardo-Cruz, J.A., Meave, J.A., Pérez-García, E.A., \& Hernández-Stefanoni, J.L. (2010). Spatial structure of plant communities in a complex tropical landscape: implications for $\beta$-diversity, Community Ecology, $11,202-210$.

Gentry, A. H. (1995). Diversity and floristic composition of neotropical dry forests. In S. H. Bullock, Mooney H. A., \& Medina. E. (Eds.), Seasonally dry tropical forests (pp. 146-190). Cambridge: University Press.

Gillespie, T. W., Grijalva, A., \& Farris, C. N. (2000). Diversity, composition, and structure of tropical dry forests in Central America. Plant Ecology, 147, 37-47.

Hernández-Ramírez, A. M., \& García-Méndez, S. (2015). Diversidad, estructura y regeneración de la selva tropical estacionalmente seca de la Península de Yucatán , México. Revista de Biología Tropical, 63(3), 603-616.

INE (Instituto Nacional de Ecología). (2000). Áreas naturales protegidas de México con decretos federales (1899-2000). México, D.F.: SEMARNAT, PNUD.

INECOL (Instituto de Ecología, A.C.). (2016). Flora de Veracruz. Recuperado de https//:www1.inecol.edu. $\mathrm{mx}$. florver/inicio/htm

INEGI (Instituto Nacional de Estadística y Geografía). (2013). Conjunto de datos vectoriales de uso del suelo y vegetación escala 1:250 000, serie V. México. INEGI. Recuperado de http://www.inegi.org.mx/geo/ contenidos/recnat/usosuelo/

Jiménez-Ramírez, J., \& Martínez-Gordillo, M. (2004). Euphorbiaceae. In A. J. García-Mendoza, M. J. Ordóñez, \& M. Briones-Salas (Eds.). Biodiversidad de Oaxaca (pp. 227-237). México: UNAM, Fondo Oaxaqueño para la Conservación de la Naturaleza, WWF.

Kovach, W. L. (2013). MVSP-A Multivariate Statistical Package for Windows, v. 3.2. Recuperado de https:// www.kovcomp.co.uk/mvsp/

Leiva, J. A., Rocha, O. J., Mata, R., \& Gutiérrez-Soto, M.V. (2009). Cronología de la regeneración del bosque tropical seco en Santa Rosa, Guanacaste, Costa Rica. La vegetación en relación con el suelo. Revista de Biología Tropical, 57, 817-836.

López-Gómez, A. M., \& Williams-Linera, G. (2006). Evaluación de métodos no paramétricos para la estimación de riqueza de especies de plantas leñosas en 
cafetales. Boletín de la Sociedad Botánica de México, $78,7-15$.

Lott, E. J., Bullock, S. H., \& Solis-Magallanes, A. J. (1987). Floristic diversity and structure of Upland and Arroyo Forests of coastal Jalisco. Biotropica, 19, 228-235.

Madrid, L., Núñez, J.M., Quiroz, G., \& Rodríguez, Y. (2009). La propiedad social forestal en México. Investigación ambiental, 1, 179-96.

Magurran, A. E. (2004). Measuring Biological Diversity. Oxford: Blackwell Publishing.

Matteucci, S. D., \& Colma, A. (1982). Metodología para el estudio de la vegetación. Washington D.C.: Organización de los Estados Americanos.

Meave, J.A., M. A., Romero-Romero, S. H. Salas-Morales, E. A. Pérez-García, \& J. A. Gallardo-Cruz. (2012). Diversidad, amenazas y oportunidades para la conservación del bosque tropical caducifolio en el estado de Oaxaca, México. Ecosistemas, 21, 85-100.

Mendoza, A. J. G., \& Meave, J. A. (2011). Diversidad florística de Oaxaca: de musgos a angiospermas, colecciones y lista de especies. Instituto de Biología Universidad Nacional Autónoma de México: Comisión Nacional para el Conocimiento y Uso de la Biodiversidad.

Merino, L. (2008). Conservación comunitaria en la cuenca alta del Papaloapan, Sierra Norte de Oaxaca. Nueva Antropología, 68, 37-49.

Miles, L., Newton, A.C., DeFries, R. S., Ravilious, C., May, I., Blyth, S., Kapos, V., \& Gordon, J. E. (2006). A global overview of the conservation status of tropical dry forests. Journal of Biogeography, 33, 491-505.

Miranda, F., \& Hernández-X. E. (1963). Los tipos de vegetación de México y su clasificación. Boletín de la Sociedad Botánica de México, 28, 29-179.

Mizrahi, A., Ramos, P. J., \& Jiménez-Osornio, J. (1997). Composition, structure and management potential of secondary dry forest in two abandoned henequen plantations of Yucatan, Mexico. Forest Ecology and Management, 96, 272-282.

Moreno, C. (2001). Métodos para medir la biodiversidad. M\&T-Manuales y Tesis SEA, vol. 1. Zaragoza: CYTED, ORCYT/UNESCO, SEA.

Moreno, C. E., Barragán, F., Pineda, E., \& Pavón, N. P. (2011). Reanálisis de la diversidad alfa: Alternativas para interpretar y comparar información sobre comunidades ecológicas. Revista Mexicana de Biodiversidad, 82(4), 1249-1261.
Mueller-Dombois, D., \& Ellenberg, H. (1974). Aims and methods of vegetation Ecology. New York: John Wiley and Sons.

Murphy P. G., \& Lugo A. E. (1995). Dry forest of Central America and the Caribbean. In S. H. Bullock, H. A. Mooney, \& E. Medina (Eds.), Seasonally dry tropical forests (pp. 9-34). Cambridge: University Press.

Murphy, P. G., \& Lugo, A. E. (1986). Ecology of Tropical Dry Forest. Annual Review of Ecology and Sistematics, 17, 67-88.

Neelo, J., Teketay, D., Kashe, K., \& Masamba, W. (2015). Stand structure, diversity and regeneration status of woody species in open and exclosed dry woodland sites around Molapo farming areas of the Okavango Delta, Northeastern Botswana. Open Journal of Forestry, 5, 313-328.

Osorio, B. M. (2012). Origen, evolución y ecología de la selva seca. Inventio, la génesis de la cultura universitaria en Morelos, México, 16, 61-69.

Palacios-Wassenaar, O., Castillo-Campos, G., VázquezTorres, S. M., \& Del Amo-Rodríguez, S. (2014). Flora vascular de la selva mediana subcaducifolia del centro de Veracruz, México. Revista Mexicana de Biodiversidad, 85, 125-142.

Pennington, T. D., \& Sarukhán, J. (2005). Árboles tropicales de México: Manual para la identificación de las principales especies. México, D.F: Fondo de Cultura Económica.

Pérez, A. M., Sotelo, M., Ramírez, F., Ramírez, I., López, A., \& Siria, I. (2006). Conservación de la biodiversidad en sistemas silvopastoriles de Matiguás y Rio Blanco (Matagalpa, Nicaragua). Ecosistemas, $15,125-141$.

RAN. (2016). El 78\% de la superficie de Oaxaca son ejidos y comunidades. México: Registro Agrario Nacional.

Reyes, J. A., Gómez, J. P., Muis, R.O., Zavala, R., Ríos, G.A., \& Villalobos, O. (2012). Atlas de Propiedad Social y Servicios Ambientales en México. México: Instituto Interamericano de Cooperación para la Agricultura, Cooperación Técnica Registro Agrario Nacional.

Rincón, E., Álvarez, A. M., González, D. G., Huante, P., \& Hernández, R. (1999). Restauración en Selvas Bajas Caducifolias. Gaceta ecológica (53), 62-71.

Rocha, L. A. (2010). Determinantes ambientales de la diversidad arbórea en la Depresión Central de Chiapas (Tesis de maestría, El Colegio de la Frontera Sur). Recuperado de http://aleph.ecosur.mx:8991/exlibris/ aleph/a22_1/apache_media/9FR7L9DXQ9HFDXBY V27JV92S82QL2X.pdf 
Rzedowski, J. (1991). Diversidad y orígenes de la flora fanerógama de México. Acta Botánica Mexicana, 14, 3-21.

Rzedowski, J. (2006). Vegetación de México. México. 1era. Edición digita. Comisión Nacional para el Conocimiento y Uso de la Biodiversidad.

SEMARNAT. (2016). Prontuario Estadístico y Geográfico de las Áreas Naturales Protegidas de México. México. Recuperado de http://entorno.conanp.gob.mx/ docs/PRONTUARIO-ANP-2015.pdf

Shackleton, C. M. (2000). Comparison of plant diversity in protected and communal lands in the Bushbuckridge lowveld savanna, South Africa. Biological Conservation, 94, 273-285.

Sneath, P. H., \& Sokal, R. R. (1973). Numerical Taxonomy. The Principles and Practice of Numerical Classification. San Francisco: Freeman.

Trejo, I. (1999). El clima de la selva baja caducifolia. Investigaciones Geográficas, 39:40-52.
Trejo, I. (2005). Análisis de la diversidad de la selva baja caducifolia en México. In G. Halffter, J. Soberón, P. Koleff \& A. Melic (Eds.), El significado de las diversidades alfa, beta y gama (pp. 111-122). Zaragoza, España: Monografias Tercel Milenio.

Trejo, I. (2010). Las selvas secas del Pacífico mexicano. En: Ceballos, G., Martínez, L., García, A., Espinoza, E., Bezaury, J., Dirzo, R. (Eds.), Diversidad, amenazas y áreas prioritarias para la conservación de las selvas secas del pacífico de México (pp. 41-51). México: CONABIO y Fondo de Cultura Económica.

Trejo, I., \& Dirzo, R. (2002). Floristic diversity of Mexican seasonally dry tropical forests. Biodiversity and Conservation, 11, 2063-2084.

W3 TROPICOS. (2016). Base de Datos VAST Del Missouri Botanical Garden. Recuperado de http://www. tropicos.org/

Williams-Linera, G., \& Lorea, F. (2009). Tree species diversity driven by environmental and anthropogenic factors in tropical dry forest fragments of central Veracruz, Mexico. Biodiversity and Conservation, 18, 3269-3293. 